\title{
Inferences from the Distributions of Fast Radio Burst Pulse Widths, Dispersion Measures and Fluences
}

\author{
J. I. Katz \\ Department of Physics and McDonnell Center for the Space Sciences \\ Washington University, St. Louis, Mo. 63130 \\ katz@wuphys . wustl .edu
}

\begin{abstract}
The widths, dispersion measures, dispersion indices and fluences of Fast Radio Bursts (FRB) impose coupled constraints that all models must satisfy. The non-monotonic dependence of burst widths (after deconvolution of instrumental effects) on dispersion measure excludes the intergalactic medium as the location of scattering that broadens the FRB in time. Temporal broadening far greater than that of pulsars at similar high Galactic latitudes implies that scattering occurs close to the sources, where high densities and strong turbulence or heterogeneity are plausible. FRB energetics are consistent with supergiant pulses from young, fast, high-field pulsars at cosmological distances. The distribution of FRB dispersion measures is: 1) Inconsistent with that of expanding clouds (such as SNR); 2) Inconsistent with space-limited source populations (such as the local Supercluster); 3) Consistent with intergalactic dispersion of a homogeneous source population at cosmological distances. Finally, the FRB $\log N-\log S$ relation also indicates a cosmological distribution, aside from the anomalously bright Lorimer burst.
\end{abstract}

Subject headings: radio continuum: general — intergalactic medium — plasmas — scattering - supernova remnants

\section{Introduction}

Following an initial report of a fast radio burst by Lorimer, et al. (2007), Thornton, et al. (2013) discovered four additional events whose large dispersion measures (DM $>500 \mathrm{pc} \mathrm{cm}^{-3}$ ) and high Galactic latitudes $|b|>40^{\circ}$ indicated that their sources were at cosmological distances. Subsequently, more FRB were discovered (the present total is ten). Their measured parameters include fluence, dispersion measure (DM) and pulse widths.

Several FRB have dedispersed and deconvolved pulse widths $W_{1300}$ at $1300 \mathrm{MHz}$ of several ms, while only upper limits were found for others. Fitting $W \propto \nu^{\beta}$ for FRB with measured $W$, their scattering indices $\beta \approx-4$, consistent with theoretical values for multipath propagation spreading in 
a plasma medium (Williamson 1972; Lee \& Jokipii 1975; Rickett 1977) and apparently confirming that the burst widths are not instrumental artefact: 1 . In homogeneous Kolmogorov turbulence $\beta=-4.4$, consistent with all values of $\beta$ measured for FRB, but inconsistent with many pulsar data (Krishnakumar, et al. 2015$)$.

This paper explores the implications of the distributions of FRB scattering widths, fluences and dispersion measures and the assumption that the dispersion measures indicate cosmological distances. Any explanation of these observations must first account for two facts: (1) All FRB have dispersion measures within a range of a factor of about three (two if the Lorimer burst is not accepted as an FRB), implying a general property, not an unusual circumstance such as a line of sight that happens to intersect a rare dense cloud; (2) The dispersion index $\alpha$, defined by the dispersive delay relation $\Delta t \propto \nu^{\alpha}$, is very close to -2 and consistent with exactly -2 for every FRB, implying an upper bound on the density of the dispersing plasma and (combined with the measured DM) a lower bound on its size. These facts are readily accounted for if most of the dispersion is intergalactic, in which case the conventional assumptions that most of the cosmic baryon density is ionized and homogeneous permits the inference of the source redshifts $z$ from the observed DM.

Searches for FRB typically impose a minimum DM of $200 \mathrm{pc} \mathrm{cm}^{-3}$ as a filter against local interference and a maximum DM of $2000 \mathrm{pc} \mathrm{cm}^{-3}$ because of pulse smearing in finite-width receiver filters, so that the absence of observed FRB DM outside the range 200-2000 pc cm${ }^{-3}$ does not imply their non-existence. Galactic FRB at high latitude would be essentially unobservable because of the low DM cutoff, unless they are surrounded by dense ionized matter, and FRB with cosmological redshift $z \geq 1.4$ are not observable because of the high DM cutoff.

The frequency-dependent temporal broadening poses a different problem. It is difficult to attribute it to intergalactic space or to our Galaxy at the high Galactic latitudes of eight of the ten reported FRB because much lower upper bounds can be placed on the broadening of at least some Galactic pulsars (Soglasnov, et al. 2004; Hankins \& Eilek 2007). This suggests that the broadening originates near the sources of the FRB. If their hosts resemble the galaxies known to us, they would be expected to contribute (unless fortuitously edge-on spirals) only a small fraction of either the broadening or the dispersion. Whatever the nature of the compact objects making FRB, it is implausible that the properties of their host galaxies differ by an order of magnitude from those of galaxies known to us. This suggests that the pulse broadening occurs in an immediate environment of the FRB that may differ from the general interstellar medium of the host galaxies (and of our own).

The Table summarizes the published data on FRB. Section 3 discusses the origin of the broadening of some FRB during propagation. Section 4 considers a specific FRB model, supergiant pulses from fast, young, high-field pulsars. This leads to consideration of young SNR as the possible origin

\footnotetext{
${ }^{1}$ Incomplete dedispersion of a burst might masquerade as broadening with $\beta=-3$ because the propagation delay $\delta \Delta t \propto(\delta \omega)^{-3}$ over a narrow frequency band $\Delta \omega$, but $\beta=-3$ is outside the measured range of uncertainty of at least one FRB (Thornton, et al. 2013).
} 


\begin{tabular}{|c|c|c|c|c|c|}
\hline FRB & $\mathrm{DM}_{E}\left(\mathrm{pccm}^{-3}\right)$ & $b$ & $S(\mathrm{Jy} \mathrm{ms})$ & $W_{1300}(\mathrm{~ms})$ & Reference \\
\hline 010621 & 213 & -4.0 & 4.3 & $<3$ & Keane, et al. (2012) \\
\hline 010724 & 350 & -41.8 & 150 & 6.2 & Lorimer, et al. (2007) \\
\hline 121102 & 369 & -0.2 & 1.2 & $<0.5$ & Spitler, et al. (2014b) \\
\hline 120127 & 521 & -66.2 & 0.8 & $<1.1$ & Thornton, et al. (2013) \\
\hline 140514 & 528 & -54.6 & 1.3 & 1.9 & Petroff, et al. (2015a) \\
\hline 110626 & 677 & -41.7 & 0.9 & $<1.4$ & Thornton, et al. $(\overrightarrow{2013})$ \\
\hline 010125 & 680 & -20.0 & 5.6 & 5 & Burke-Spolaor \& Bannister (2014) \\
\hline 131104 & 710 & -22.2 & 2.3 & 4 & Ravi, Shannon \& Jameson $(2015)$ \\
\hline 110220 & 910 & -54.7 & 7.3 & 5.6 & Thornton, et al. (2013) \\
\hline 110703 & 1072 & -59.0 & 1.8 & $<4.3$ & Thornton, et al. $(\overline{2013})$ \\
\hline
\end{tabular}

Table 1: Summary of FRB data, ordered by estimated extra-Galactic dispersion measures $\mathrm{DM}_{E}$; for the two low- $b$ (Galactic latitude) bursts these may be uncertain and are italicized. Most data are from references in the table, but fluences $S$ are from the re-evaluation by Keane \& Petroff (2015), except for the "Lorimer burst" (FRB 010724) and FRB 131104. Pulse widths $W_{1300}$ are from the references after dedispersion and deconvolution of the instrumental response and scaled, if necessary, $\propto \nu^{-4}$ to $1300 \mathrm{MHz}$ for consistency with Thornton, et al. (2013) (scalings are over modest frequency ratios and insensitive to the scaling exponent).

of FRB dispersion, in which case FRB distances would not be cosmological. Dispersion local to the FRB is discussed in Section 5. SNR are rejected on the basis of the distribution of dispersion measures, but massive quasi-static clouds are not. Section 6 constrains the number of active FRB sources, while Section 7 compares this to the supernova rate. Section 8 uses the $\log N-\log S$ relation to constrain the space distribution of FRB. The hypothesis of cosmological distances inferred from the dispersion measures of FRB is tested in Section 9 and found to fit the distribution of dispersion measures well. Section 10 contains a summary discussion. Appendix A argues that FRB are unlikely to be chirped radar pulses.

\section{Bounds Implied by Dispersion Indices}

The dispersion index $\alpha$, defined by the dispersion delay $\Delta t \propto \nu^{\alpha}$, is a strong constraint on the density of the dispersing plasma. The tightest constraint is for FRB 140514 for which $\alpha=-2.000 \pm 0.004$ (Petroff, et al. 2015a); all measured values of $\alpha$ are consistent with -2 , though others have somewhat larger uncertainties. Expansion of the dispersion relation for electromagnetic waves in a cold (nonrelativistic) plasma in powers of $\omega_{p}^{2} / \omega^{2} \ll 1$ yields Katz $2014 \mathrm{~b}$ )

$$
\Delta t=\int \frac{d \ell}{c} \frac{1}{2} \frac{\omega_{p}^{2}}{\omega^{2}}\left(1+\frac{3}{4} \frac{\omega_{p}^{2}}{\omega^{2}}+\cdots\right)
$$


and

$$
\alpha \equiv \frac{d \ln \Delta t}{d \ln \omega}=-2-\frac{3}{2} \frac{\omega_{p}^{2}}{\omega^{2}}+\cdots=-2-\frac{6 \pi n_{e} e^{2}}{m_{e} \omega^{2}}+\cdots .
$$

If dispersion occurs at a significant redshift then $\omega$ is the frequency in the region in which the dispersion takes place (blue-shifted from the frequency of observation) and the plasma frequency $\omega_{p}$ refers to the electron density there (Dolag, et. al. 2015).

In order to constrain $n_{e}$ in the scattering region we must allow for the fact that it contributes only $\mathrm{DM}_{\text {scatt }}$ to the (extra-Galactic) dispersion of the pulse. The remainder, perhaps nearly all, is attributed to intergalactic propagation, for which the $\omega_{p}^{2} / \omega^{2}$ and higher terms in 22 are negligible. From the observed bounds on $\alpha,(2)$ yields

$$
\frac{\omega_{p}^{2}}{\omega^{2}} \frac{\mathrm{DM}_{\text {scatt }}}{\mathrm{DM}} \leq \frac{2}{3} \max (-\alpha-2) \lesssim 0.003,
$$

where $\max (-\alpha-2) \approx 0.004$ is the observed upper bound on $-\alpha-2$ for FRB 140514 (Petroff, et al. 2015a). Using $\omega_{p}^{2}=4 \pi n_{e} e^{2} / m_{e},(2)$ and (3),

$$
n_{e} \lesssim \frac{1}{6 \pi} \frac{m_{e} \omega^{2}}{e^{2}} \max (-\alpha-2) \approx 1 \times 10^{8} \mathrm{~cm}^{-3} .
$$

The size $R$ of the dispersing region is bounded:

$$
R=\frac{\mathrm{DM}}{n_{e}} \gtrsim 2 \times 10^{13} \mathrm{~cm} .
$$

This temperature-independent limit excludes models that attribute the dispersion to the immediate environment of a star.

Analogous but temperature-dependent limits are implied by the requirement that the dispersing cloud be transparent to inverse bremsstrahlung absorption. The inverse bremsstrahlung limits scale $\propto T^{-3 / 2}$ and are relaxed if absorption of an energetic pulse heats the plasma.

\section{Pulse Widths}

We approximate the burst propagation as that in a flat static (Euclidean) universe. For the estimated redshifts $z \leq 0.96$ inferred from the dispersion measures by attributing the dispersion to intergalactic plasma with conventional cosmological parameters (the overwhelming majority of the baryon density distributed homogeneously in intergalactic space (Macquart, et al. 2015); possible inhomogeneity is discussed by Zhou, et al. (2014)), this only introduces an error of a factor $\mathcal{O}(1)$, less than other uncertainties.

Following the classic model of Williamson (1972); Lee \& Jokipii (1975); Rickett (1977), we approximate the propagation paths as produced by a single scattering at a distance $a D$ from us

and $(1-a) D$ from the source. If the scattering angle $\Delta \theta \ll 1$ then the angles $\phi \approx(1-a) \Delta \theta$ and $\chi \approx a \Delta \theta ;$ the geometry is shown in Fig. 1 . 


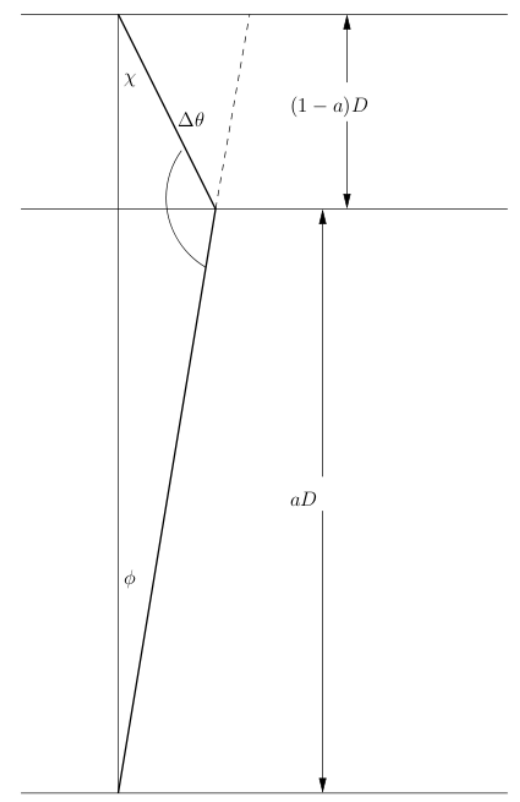

Fig. 1. - Path of scattered radiation (Williamson 1972, Kulkarni, et al. 2014)

We assume that the origin of the pulse width $W$ is dispersion in propagation path lengths. The incremental delay attributable to scattering by an angle $\Delta \theta$ (Williamson 1972; Kulkarni, et al. 2014) is

$$
W \approx \frac{D}{2 c}(\Delta \theta)^{2} a(1-a)
$$

Then

$$
\Delta \theta \approx \sqrt{\frac{2 c W}{D a(1-a)}} \gtrsim \sqrt{\frac{8 c W}{D}} \sim 5 \times 10^{-10},
$$

for all FRB with measured $W$. The minimum value is obtained for $a=1 / 2$. The angular width of the received radiation

$$
\phi \approx(1-a) \Delta \theta=\sqrt{\frac{2 c W}{D}\left(\frac{1-a}{a}\right)} .
$$

Because of its low density, the intergalactic medium is unlikely to be the location of scattering. The Table shows that the pulse widths $W_{1300}$ do not vary monotonically with dispersion measure, in contrast to expectations for intergalactic scattering (Macquart \& Koay 2013). In addition, Luan \& Goldreich (2014) have shown that Kolmogorov turbulence in the intergalactic medium cannot explain the pulse broadening because the implied dissipation of turbulent energy would be excessive and the turbulence would decay rapidly (this argument does not apply to quasi-static isobaric heterogeneities such as the multi-phase structure of the interstellar medium, and its possible intergalactic analogues). 
If a scattering screen is close to the source $(1-a \ll 1)$ or to the observer $(a \ll 1)$ then $W \propto D \min (a, 1-a)$. The pulse broadening produced by Galactic scattering $(a \ll 1)$ is comparable to that of a Galactic pulsar in the same direction because the screen's distance $a D$ is similar, even though $D$ may be seven orders of magnitude greater for the FRB, while $1-a=\mathcal{O}(1)$ for both the Galactic pulsar and the distant FRB.

Galactic pulsars at high $b$ show orders of magnitude less pulse broadening than FRB (Krishnakumar, et al. 2015). Even at low $b$, the nanoshots of the Crab pulsar (Hankins \& Eilek 2007) imply, assuming $\nu^{-4}$ scaling from $9.25 \mathrm{GHz}$, broadening $\lesssim 1.0 \mu$ s at $1300 \mathrm{MHz}$ and those of PSR $\mathrm{B} 1937+21$, scaling only from $1.65 \mathrm{GHz}$ (hence insensitive to the assumed scaling relation), broadening $\lesssim 40$ ns (Soglasnov, et al. 2004). Unless these are the consequence of focusing, with the same propagation time for all focused paths by Fermat's principle, they indicate that Galactic scattering cannot contribute significantly to the pulse widths of FRB.

Excluding intergalactic and Galactic scattering as the origin of the pulse broadening implies it takes place near the FRB sources. $\Delta \theta$ must be orders of magnitude larger than the numerical value in Eq. 7 because $1-a \ll 1$. We know nothing of the host galaxies of FRB, but galaxies at $z \leq 1$ roughly resemble local galaxies. By the preceding argument, their general interstellar media cannot contribute a major fraction of the pulse broadening (or of the dispersion measure) unless all the five (four if the Lorimer burst is excluded) FRB with reported pulse broadening are found in spirals viewed edge-on, a statistical improbability.

The immediate environment of the FRB must be much more strongly scattering than ordinary interstellar media, indicating a causal relation with the FRB itself. FRB are likely to be young objects because compact, strongly scattering, gas clouds would quickly dissipate (Section 5.1) if unbound.

\section{Pulsar Super-Pulses?}

There is an obvious resemblance between FRB and the giant pulses of some radio pulsars. Like radio pulsars, FRB are coherent emitters (Lorimer, et al. 2007; Thornton, et al. 2013; Katz 2014a) with very high brightness temperatures. Could FRB be the same phenomenon on a more energetic scale, perhaps triggered by collapse of the neutron star (Falcke \& Rezzolla 2014)?

The high dispersion measures (and high Galactic latitudes) of FRB indicate cosmological distances and much greater powers than inferred for Galactic pulsars; Thornton, et al. (2013) estimated, assuming isotropic emission, $P \gtrsim \mathcal{O}\left(10^{42}\right) \mathrm{erg} / \mathrm{s}$ for FRB 110220 , where the lower limit assumes that the observed pulse width is partly intrinsic and not entirely the result of scattering. This should be compared to the classic result for a rotation-powered pulsar's spindown power (converted to radio emission with an efficiency that is $\ll 1$ for all known radio pulsars)

$$
P_{\text {spindown }} \approx \frac{2 \mu^{2} \Omega^{4}}{3 c^{3}} \approx 2 \times 10^{44} \mu_{30}^{2} \Omega_{4}^{4} \mathrm{erg} / \mathrm{s},
$$


where $\mu_{30} \equiv \mu / 10^{30}$ gauss $\mathrm{cm}^{3}$ is the scaled magnetic dipole moment and $\Omega_{4} \equiv \Omega / 10^{4} \mathrm{~s}^{-1}$ is the scaled rotation frequency. This relation is exact for an oblique $\left(90^{\circ}\right)$ rotator in vacuum and is believed to be approximately correct for any angle between magnetic and rotational axes. The angular distribution of this power is unknown; dipole emission and winds from spindown of aligned rotors are roughly isotropic, but how this power is converted to coherent $\mathrm{GHz}$ radio emission, and its resulting angular distribution, is not understood.

Eq. 9 indicates that a combination of high dipole moment and fast spin would be required to explain FRB as rotation-powered pulsars. Radio pulsars are known with such high values of $\mu$ and $\omega$, but not in combination. The combination, if it occurs, would lead to a short spin-down time

$$
t_{\text {spindown }}=\frac{1}{2} \frac{I \Omega^{2}}{P_{\text {spindown }}}=\frac{3}{4} \frac{I c^{3}}{\mu^{2} \Omega^{2}} \approx \frac{10 \mathrm{y}}{\left(\mu_{30} \Omega_{4}\right)^{2}} \lesssim 10^{3} \Omega_{4}^{2} \mathrm{y},
$$

where $I \sim 10^{45} \mathrm{~g} \mathrm{~cm}^{2}$ is the moment of inertia and the last inequality uses Eq. 9. For a neutron star spinning near breakup and $P_{\text {spindown }}$ as large as required by a cosmological distance for FRB 110220 (Thornton, et al. 2013), $t_{\text {spindown }}$ could be as long as $\sim 10^{3} \mathrm{y}$, but this would require implausibly efficient emission and optimal choice of parameters.

\subsection{Soft Gamma Repeaters?}

A fundamental property of rotation-powered pulsar models is that their instantaneous power (including that into a particle wind that is believed to dominate the energetics) is limited by Eq. 9 . there is no intermediate energy reservoir between the rotation and accelerated particles or radiated fields that could be drawn down in sudden bursts of higher power. Rotation-powered pulsar models are thus distinguished from "magnetar" models (Katz 1982, Thompson \& Duncan 1992; Mereghetti 2008) of soft gamma repeaters (SGR) powered by dissipation of magnetic energy. The upper limit to the rate of energy release in a magnetar model is set by the poorly understood process of magnetic reconnection, and might be as high as $\mathcal{O}\left(R_{N S}^{2} B^{2} c\right) \sim 10^{46} \mu_{30}^{2} \mathrm{erg} / \mathrm{s}$, as required to account for the December 27, 2004 outburst of SGR 1806-20, assuming isotropic emission, as would be expected for a magnetically trapped pair plasma.

In SGR this power is thermalized, appearing as roughly black body emission of soft gamma rays or hard X-rays, not as the high brightness radio frequency emission or relativistic particle acceleration of rotation-powered pulsars; thermalization may be inevitable at intensities $\gtrsim 10^{29}$ $\mathrm{erg} / \mathrm{cm}^{2} \mathrm{~s}^{-1}$ Katz 1996). Perhaps the same object might be both a SGR and an FRB (Kulkarni, et al. 2014), though at different times or at different places in its magnetosphere. For example, PSR J1745-2900 is observed both as an anomalous X-ray pulsar (and hence is often termed a magnetar; Mori, et al. (2013)) and as a radio pulsar (Eatough, et al. 2013) emitting high-brightness nonthermal radiation.

A Galactic FRB, if above the horizon, could have been detected in the far sidelobes of any radio telescope observing in the L-band (Katz 2014a with sufficient time resolution to detect a 
transient. No such detections of the known giant outbursts of Galactic SGR have been reported, but it is unclear if any radio telescope was operating in a suitable mode, if the SGR were above the horizon, or if such a signal would have been rejected as interference.

\section{Dispersion Local to FRB?}

\subsection{Distribution of Dispersion Measures in Young SNR}

Some of the difficulties of the pulsar supergiant-pulse hypothesis would be mitigated if the dispersion were produced in the pulsar's immediate vicinity, so that the DM would not imply cosmological distances, reducing the required energy (Pen \& Connor 2015, Connor, Sievers \& Pen 2015). Rotation-powered pulsar models of FRB imply short-lived, and hence young, sources, but the constraints of Section 4 would be mitigated if they are cosmologically local. If so, a surrounding young SNR might be the chief source of dispersion (Kulkarni, et al. 2014; Connor, Sievers \& Pen 2015), possibly with a major Galactic contribution for the two low-latitude FRB. The local dispersion measure of a source at the center of a spherical cloud of ionized gas of mass $M$ and radius $R$ is

$$
\mathrm{DM}_{\text {local }}=818 \frac{M}{M_{\odot}}\left(\frac{R}{0.1 \mathrm{pc}}\right)^{-2} f \mathrm{pc} \mathrm{cm}^{-3},
$$

where $f=1$ for a homogeneous sphere and $f=1 / 3$ for a thin shell, implying $R \sim 0.1$ pc for a SNR, lost stellar envelope, etc. providing much of the dispersion measure of an FRB.

As discussed in Section 3, this cloud must explain most of the pulse broadening, whether or not it contributes most of the dispersion measure. If the customary Kolmogorov turbulence model of diffractive scattering (Rickett 1977) is assumed, then

$$
C_{n_{e}}^{2} \approx 0.5\left(\frac{W}{5 \mathrm{~ms}} \frac{1 \mathrm{Gpc}}{D}\right)^{5 / 6}\left(\frac{0.1 \mathrm{pc}}{R}\right) \mathrm{m}^{-20 / 3} .
$$

This is comparable to values found for the more distant and highly dispersed Galactic pulsars (Krishnakumar, et al. 2015), although the measured pulsar $C_{n_{e}}^{2}$ range over orders of magnitude. Lewandowski, et al. (2013) have shown that the scattering spectral indices of many pulsars are far from the Kolmogorov model prediction of -4.4 , and there is no a priori reason to expect either this model or values of $C_{n_{e}}^{2}$ found for the Galactic interstellar medium to be applicable to the environments of FRB.

The characteristic lifetime $T$ of an expanding cloud

$$
T \approx \frac{R}{V} \approx 30 \frac{R}{0.1 \mathrm{pc}} \frac{3000 \mathrm{~km} / \mathrm{s}}{V} \mathrm{y} \approx 30 \sqrt{\frac{f}{\mathrm{DM}_{1000}} \frac{M}{M_{\odot}}} \frac{3000 \mathrm{~km} / \mathrm{s}}{V} \mathrm{y},
$$

where $V$ is the expansion velocity and $\mathrm{DM}_{1000} \equiv \mathrm{DM}_{\text {local }} /\left(1000 \mathrm{pc} \mathrm{cm}^{-3}\right)$. At $R=0.1 \mathrm{pc}$ only $\sim 10^{-4} n_{I S M} M_{\odot}$ of interstellar material will have been swept up, for an interstellar density of $n_{I S M}$ 
atoms $/ \mathrm{cm}^{3}$, so $V$ is nearly the initial explosion velocity. If $V$ is within the range $3000-30000 \mathrm{~km} / \mathrm{s}$ of SN ejecta then the age of the cloud $t \leq T \lesssim 30 \mathrm{y}$. If FRB are found within such clouds, then if repetitive bursts are observed their dispersion measures will decrease monotonically and smoothly according to 11 with $R=V t$. The absence of known SN within the last $\sim 30 \mathrm{y}$ at the high Galactic latitude positions of most FRB sets lower bounds on their distances.

The hypothesis that most of the dispersion is produced in an expanding cloud around the FRB also predicts the distribution of dispersion measures, subject to the unknown event rate (for example, bursts may be more frequent in younger objects, or may not occur until after a latency period from their birth). If we assume an age-independent event rate (at least over the period over which the SNR provides much of the observed dispersion measure), then from (11) and (13) we find

$$
\frac{d N}{d \mathrm{DM}} \propto \mathrm{DM}^{-3 / 2}
$$

In Figure 2 we show the cumulative distribution of dispersion measures of the high latitude FRB shown in the Table, and compare to the relation $N \propto \mathrm{DM}^{-1 / 2}$ predicted by Eq. 14 . Even though only eight such FRB are known, their cumulative distribution is robust. The fit is not good.

The observed distribution of DM does not display the long "tail" of high DM bursts predicted by cosmologically local models in which much of the dispersion is provided by an expanding gas cloud such as (but not necessarily) a SNR. Nor does it show the predicted divergence at low DM. Even if the absence of this low-DM divergence were explained by short active lifetimes of the FRB sources, as required by Eq. 10 and consistent with Eq. 13 , the deficiency of high DM FRB argues against the hypothesis that most of the dispersion is provided by expanding SNR (Kulkarni, et al. 2014: Connor, Sievers \& Pen 2015). It therefore argues in favor of the cosmological distances inferred by attributing the dispersion measures to the intergalactic medium. Then a deficiency of high DM FRB may be attributed to the lesser fluence (greater luminosity distance) of more distant FRB.

This does not require rejection of the hypothesis that FRB are produced by high field fast rotation powered pulsars. Attributing most of the dispersion to the intergalactic medium and accepting cosmological distances would resolve the difficulty (Eq. 13) that if a SNR is to provide the dispersion its rapid expansion (especially if $V>3000 \mathrm{~km} / \mathrm{s}$, as found for most SN) would require FRB activity to occur very soon after the pulsar's birth. However, the constraint $t<t_{\text {spindown }}$ would remain.

\subsection{Quasi-Static Clouds}

If the dispersion occurs in a stable quasi-static plasma cloud, then the condition that FRB activity occur within a time given by Eq. 13 would no longer apply. Such clouds need not be 


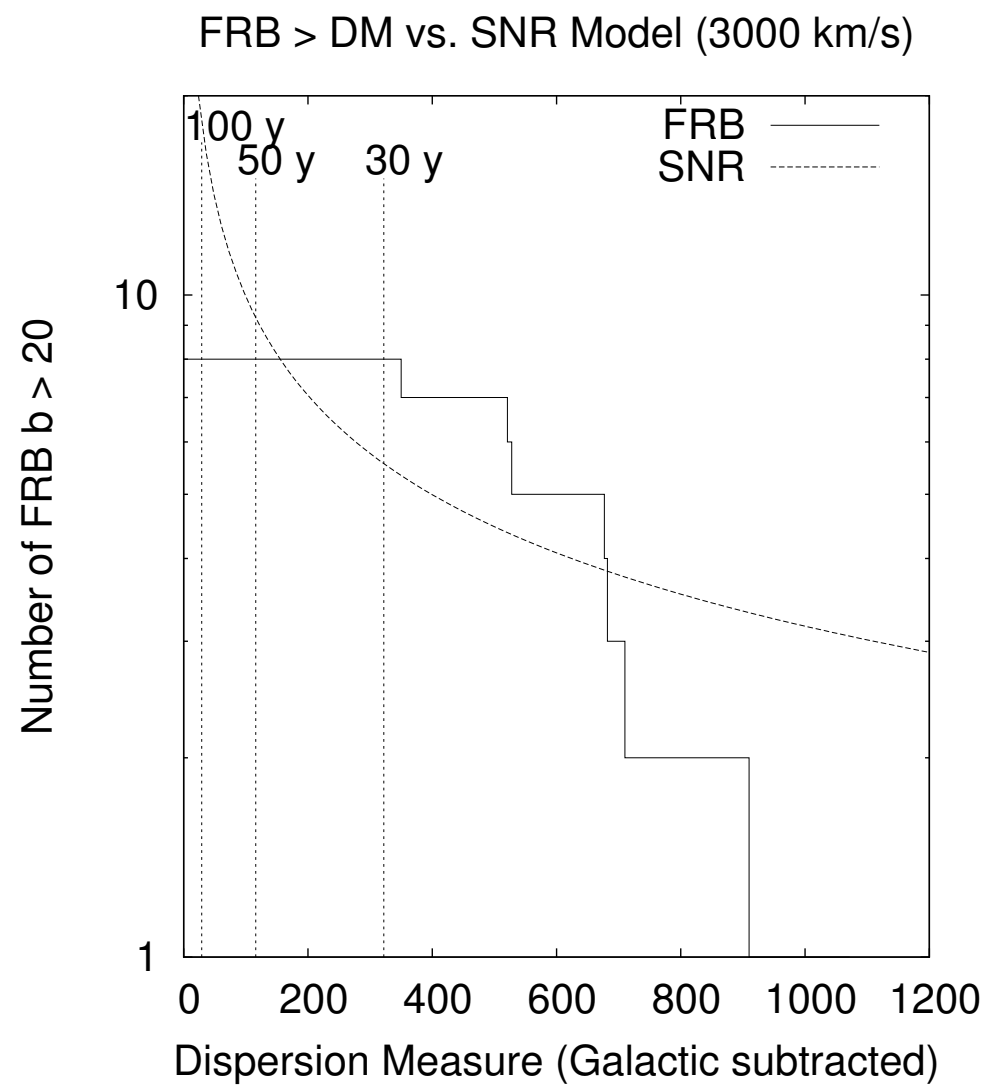

Fig. 2.- Cumulative distribution of dispersion measures of high latitude $\left(|b|>20^{\circ}\right) \mathrm{FRB}$ compared to a fitted distribution of dispersion measures of expanding SNR shells. The predicted divergence at small dispersion measures is absent, requiring an early cutoff to FRB activity. The dotted lines indicate the cutoff ages required to avoid a (not observed) divergence of the population at low DM; these ages are given for a $1 M_{\odot}$ shell with expansion speed $V=3000 \mathrm{~km} / \mathrm{s}$ and scale $\propto M^{1 / 2} / V$. The observed FRB distribution also disagrees with the prediction of the SNR hypothesis for DM $\gtrsim 700$ $\mathrm{pc}-\mathrm{cm}^{-3}$. In this model there should be no correlation between DM and brightness because DM depends only on the SNR parameters, not the distance to the FRB, so a deficiency of high DM bursts cannot be explained as a consequence of greater distance, as it might be if dispersion is intergalactic.

associated with the compact source of the FRB, but might be, for example, dense formerly molecular clouds ionized by a starburst or the ionized interstellar medium of an irregular galaxy with rapid star formation. Specific examples, found in regions that may be plausible sites of FRB, include dense ionized star-forming structures (Kulkarni, et al. 2014) and circum-galactic nuclear gas (Pen \& Connor 2015).

Eq. 13 is then replaced by the Jeans condition that the cloud be stable against gravitational 
collapse. This would constrain its parameters:

$$
\sqrt{\frac{G M}{R}} \lesssim c_{s}=\sqrt{\frac{5 k_{B} T\left(1+\mu_{e}\right)}{3 m_{p}}},
$$

where $c_{s}$ is the sound speed and $\mu_{e} \approx 0.85$ is the number of electrons per baryon. Substituting $M \approx R^{3} m_{p} n_{e} / \mu$ and $\mathrm{DM} \approx n_{e} R$ (attributing the dispersion to the source's plasma cloud, not the the intervening line of sight), we find

$$
R \lesssim \frac{5\left(1+\mu_{e}\right) \mu_{e} k_{B} T}{3 G \mathrm{DM} m_{p}^{2}} \approx 5 \times 10^{21} \frac{T_{8000}}{\mathrm{DM}_{1000}} \mathrm{~cm}
$$

and

$$
n_{e} \sim \frac{\mathrm{DM}}{R} \gtrsim 0.6 \mathrm{DM}_{1000}^{2} T_{8000}^{-1} \mathrm{~cm}^{-3}
$$

where we normalize the temperature $T_{8000} \equiv T / 8000^{\circ} \mathrm{K}$ (following Kulkarni, et al. (2014) ) and the dispersion measure $\mathrm{DM}_{1000} \equiv \mathrm{DM} / 1000 \mathrm{pc} \mathrm{cm}^{-3}$, and assume complete ionization and cosmic abundances. The corresponding mass

$$
M \lesssim \frac{25 k_{B} T\left(1+\mu_{e}\right)^{2} \mu_{e}}{9 G^{2} m_{p}^{3} \mathrm{DM}} \approx 8 \times 10^{7} \frac{T_{8000}^{2}}{\mathrm{DM}_{1000}} M_{\odot} .
$$

The hydrodynamic time

$$
T_{J} \sim \frac{R}{c_{s}} \lesssim \sqrt{\frac{5 k_{B} T\left(1+\mu_{e}\right)}{3 m_{p}}} \frac{\mu_{e}}{G m_{p} \mathrm{DM}} \approx 10^{8} \frac{T_{8000}^{1 / 2}}{\mathrm{DM}_{1000}} \mathrm{y}
$$

has no explicit dependence on the unknown parameters $n_{e}, R$ and $M . T_{J}$ is long enough to avoid the statistical problems (Section 5.1) posed by attributing the dispersion measures to young SNR, whose youth implies that only a very few are active with the observed dispersion measures at any time. The dispersive cloud could be more compact and dense than the bounds $(16)$ and (17), perhaps by a large factor.

These bounds are consistent with dense quasi-static compact clouds in the FRB neighborhood (Pen \& Connor 2015) while avoiding the rapid expansion and short lifetime implied by attributing dispersion to young SNR. Much smaller $R$ and $M$ and larger $n_{e}$ than the bounds are possible. The bounds also admit a protogalaxy or starburst ionized by an initial generation of hot luminous stars, providing the observed dispersion measures. Such sites may be plausible locales for FRB, but give no clues to the origin of the FRB themselves beyond indicating a relation with massive stars and high rates of star formation and death.

\section{How Many FRB Sources?}

Models of FRB may be divided into two general classes, those in which they are the product of catastrophic events (e.g., SN, neutron star mergers, neutron star accretion by black holes, GRB) 
that destroy their participants and cannot repeat, and non-catastrophic events (e.g., giant pulsar pulses, SGR outbursts) that can repeat. In the former case the number of sources will equal the number of observed FRB, but in the latter case repetitions may be observed. The confirmed observation of a single repetition would establish their origin in non-catastrophic events. Here we consider the constraints that can be placed on $N_{\text {sources }}$ non-catastrophic sources if, as at present, no repetitions have been observed.

There are two constraints on the number of presently active detectable FRB sources $N_{\text {sources }} \equiv$ $B T$, where $B$ is their birth rate within the volume from which FRB may be detected and $T$ is their active lifetime (during which they have the defining properties of FRB, including outbursts and dispersion measures). If the bursts occur stochastically, without any latency period following a burst, then the absence of coincidences among $N_{F R B}$ observed FRB implies

$$
N_{\text {sources }} \gtrsim N_{F R B}^{2}=100 \text {. }
$$

As more FRB are discovered, either coincidences (repetitions) will be observed, implying

$$
N_{\text {sources }} \sim \frac{N_{\text {FRB }}^{2}}{N_{\text {coincidences }}},
$$

or the lower bound of Eq. 20 will increase.

The absence of repetitions of any individual FRB implies

$$
N_{\text {sources }} \gtrsim \Omega_{F R B} \tau_{\text {min }} \sim 10^{3},
$$

where $\Omega_{F R B}$ is the all-sky FRB rate and $\tau_{\min }$ is the empirical lower bound on the repetition time of an individual source. Keane \& Petroff (2015) and Ravi, Shannon \& Jameson (2015) estimate $\Omega_{F R B} \sim 0.03 / \mathrm{s}$ with an uncertainty as large as a factor of three and $\tau_{\min } \sim 10 \mathrm{~h}$.

If the bursts are stochastic then $\tau_{\text {min }} \gtrsim \tau_{\text {tot }}$, the total time beams pointed in the known

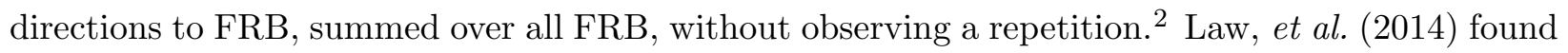
no recurrences in $1.1 \times 10^{5} \mathrm{~s}$ of observations of a single FRB, implying a $95 \%$ confidence bound $\tau_{\min }>2.7 \times 10^{4} \mathrm{~s}$, giving the numerical estimate in 22 . On the other hand, if there is a latency period between FRB from a single source then, depending on how observing time was distributed, $\tau_{\min }$ may be as short as $\tau_{\text {cont }}$, the longest duration of continuous observation of an individual FRB location without a repetition. The conditions $20-22$ may be used to test models of $N_{\text {sources }}$ against the empirical parameters $N_{F R B}, \tau_{\min }$ and $\Omega_{F R B}$, and thereby to constrain models of the sources, of their astronomical environments, and of their distances.

\footnotetext{
${ }^{2}$ It is not necessary that a beam be pointed to a single FRB for this time because, if they all have the same properties, staring in all directions in which an FRB has been observed is equivalent. It is also assumed that localization is good enough that the chance of misidentifying a new source as a repetition of a previously observed source is negligible; for $15^{\prime}$ localization and $N_{F R B} \sim 10$ this chance is $\sim 2 \times 10^{-5}$.
} 


\section{Distances from Comparison to SN Rate}

Here we consider the consequences of the plausible, but unproven, assumption that FRB are associated with SN. The number of SNR of ages $t<T$ (Eq. 13) associated with our Galaxy (out to distances $\sim 1 \mathrm{Mpc}$ ) with sufficient column density to provide the observed FRB dispersions is inferred from a Galactic SN rate of $0.03 / \mathrm{y}$ to be $N_{S N R} t<T \lesssim \mathcal{O}(1)$, inconsistent with the observation of 10 FRB. Eq. 20 provides an even stronger argument against such close, SN-associated, sources. Further, the all-sky FRB rate $\Omega_{F R B} \sim 0.03 / \mathrm{s}$ would imply a repetition time of an individual source $\tau \sim N_{\text {sources }} / \Omega_{F R B}=N_{S N R} t<T / \Omega_{F R B} \sim 30 \mathrm{~s}$. The hypothesis of such rapid repetitions of FRB is excluded empirically by orders of magnitude (Law, et al. 2014 ; Petroff, et al.|2015a).

If FRB are associated with SN, at a rate of order one-to-one (the FRB do not repeat), comparison of the rates of the two classes of events shows that their distances must be cosmological: The SN rate is estimated (Sharon, et al. 2007$)$ to be $\Omega_{S N} \approx 0.098 \times 10^{-12} M_{\odot}^{-1} \mathrm{y}^{-1}$. Standard cosmological parameters indicate a local baryon density $\rho_{\text {baryon }}=1.9 \times 10^{-64} M_{\odot} \mathrm{cm}^{-3}$ and a SN

rate $\Omega_{S N} \rho_{\text {baryon }} \approx 1.9 \times 10^{-77} \mathrm{~cm}^{-3} \mathrm{y}^{-1}$. Comparison to the all-sky FRB rate $\Omega_{F R B} \approx 0.03 / \mathrm{s}$ indicates

$$
D \sim\left(\frac{3}{4 \pi} \frac{\Omega_{F R B}}{\Omega_{S N} \rho_{\text {baryon }}}\right)^{1 / 3} \sim 1 \mathrm{Gpc} .
$$

With these assumptions FRB must originate at cosmological distances, even if much of their dispersion measures are local to their sources. Local matter might be the source of dispersion if FRB are giant pulsar pulses or SGR outbursts (Kulkarni, et al. 2014), but neither of these can explain the distribution of dispersion measures (Section 9).

If, on the other hand, many FRB are associated with each SN, as is plausible if they are supergiant pulsar pulses, we can still set a lower bound on the distance out to which FRB are observed by requiring that the number of FRB sources be at least the number of $\mathrm{SN}$ in the active lifetime $T_{F R B}$ of the product of a $\mathrm{SN}$ :

$$
D \gtrsim\left(\frac{3 N_{\text {sources }}}{4 \pi \Omega_{S N} \rho_{\text {baryon }} T_{F R B}}\right)^{1 / 3} \sim 10 \mathrm{Mpc} ;
$$

the numerical value assumes $T_{F R B} \sim 3000 \mathrm{y}$, the estimated active lifetime of a SGR. If, instead, $T_{F R B}$ is taken to be $\sim 30 \mathrm{y}$, the duration over which a SNR can provide the observed dispersion measure $(13)$, then $D \gtrsim 50 \mathrm{Mpc}$. Finally, the absence of obvious correlation with cosmologically local structure (clusters and superclusters of galaxies) suggests $D \gtrsim \mathcal{O}(100) \mathrm{Mpc}$.

\section{8. $\log \mathbf{N}-\log \mathrm{S}$}

The distribution of fluxes or fluences for a population of objects is a classic tool for determining their distribution in space. It was used in the early days of radio astronomy to exclude steady state cosmology, and in gamma-ray burst astronomy it indicated their origin at cosmological distances. 
Because $N(S)$ is the cumulative number of sources with flux or fluence greater than some threshold $S$, meaningful conclusions can be drawn from remarkably small values of $N(S)$. For FRB $S$ must be the fluence. In flat static (Euclidean) geometry an inverse square law holds and $N(S) \propto S^{-3 / 2}$. Results for real cosmological models cannot be disentangled from the evolution of the FRB event rate, which is completely unknown, so we compare to the Euclidean result, which is approximately valid for $z \lesssim 1$.

The data are shown in Fig. 3. If allowance is made for the likely incompleteness of the sample for $S<1$ Jy ms, the distribution is consistent with homogeneous Euclidean space, as also suggested by its isotropy, except for the anomalous Lorimer burst. Keane \& Petroff (2015) give only a lower bound on its fluence of $31.5 \mathrm{Jy} \mathrm{ms}$, rather than the specific (but uncertain) value of $150 \mathrm{Jy} \mathrm{ms}$ cited by Lorimer, et al. (2007) and used in the Figure, but even if this minimum is its actual value its deviation from the power law would still be problematic.

These results do not determine a distance scale, but do indicate that FRB are not limited to a bounded region. Provided the deviation from the power law at low $S$ is attributed to artefacts (for example, different research groups or instruments having differing calibrations or thresholds for detection or acceptance of transients as FRB), they argue against models in which FRB are associated with bounded structures (the Solar System, Galaxy, Local Group, Local Supercluster, local universe in which redshift and cosmic evolution are negligible, etc.) outside of which the FRB density drops. If, on the other hand, the deviation at low $S$ is real then some such structure is indicated 3

\section{Distribution of Dispersion Measures-Cosmological}

It is possible to use the distribution of FRB dispersion measures to constrain their spatial distribution if the dispersion is attributed to a uniform intergalactic medium. This is complementary to the use of the $\log N-\log S$ distribution. Unlike $\log N-\log S$, this method is specific to FRB.

The number of FRB with origin between distances of $r$ and $r+d r$

$$
d N=K(r) r^{2} d r
$$

where $K(r)$ should include the effects of the geometry of the universe, the redshift of the event rate, the evolution and varying density of the source population and the K-correction (redshift of the spectrum). The source evolution and K-correction are unknown. For low redshift $z \ll 1$

\footnotetext{
${ }^{3}$ The low fluence deviation cannot be explained by the variation of sensitivity across a beam. The reported fluences are nominal values that apply if the FRB is centered in the beam, and are not corrected for the beam pattern (a correction that cannot be made because the location of the FRB within the beam is not known, except possibly for the Lorimer burst that was detected in the sidelobes of two beams in addition to its principal detection). Each element of beam solid angle contributes a distribution $\delta N(S) \propto S^{-3 / 2}$, and their sum $N(S) \propto S^{-3 / 2}$.
} 


\section{FRB Cumulative Distribution vs. Fluence}

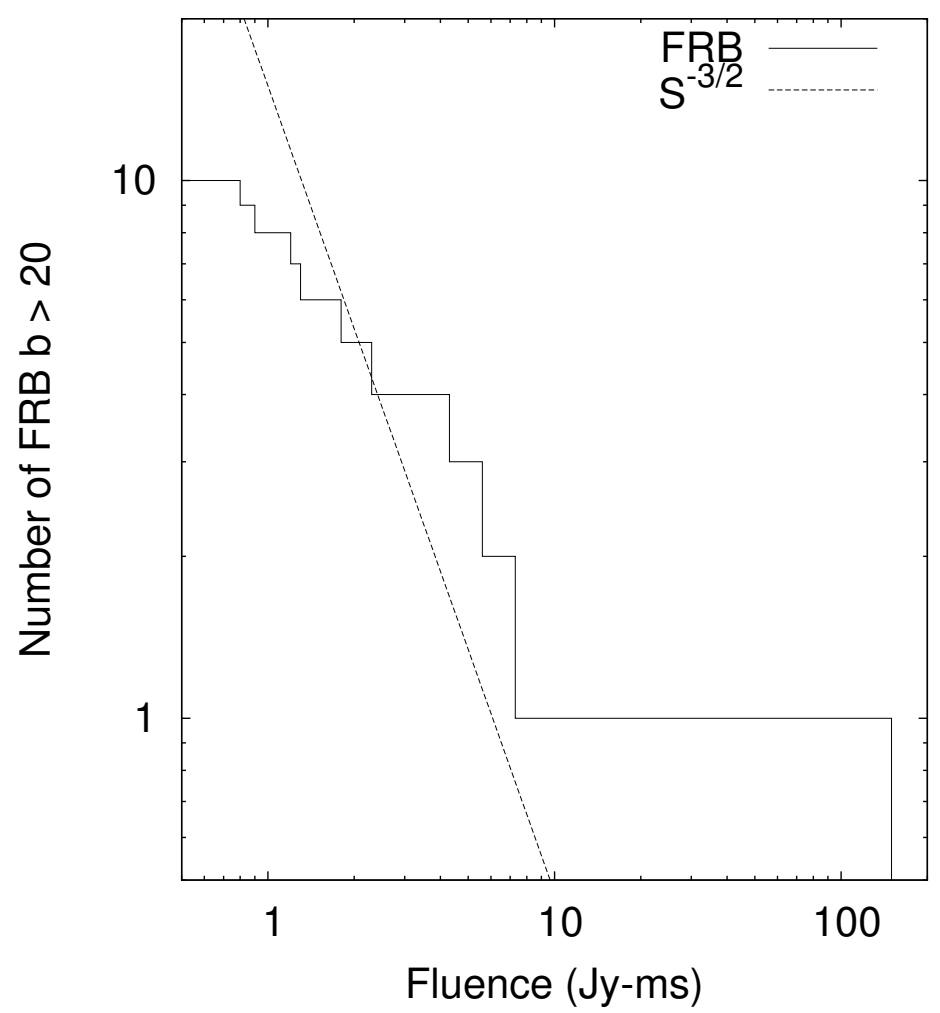

Fig. 3.- Cumulative $\log N$ vs. $\log S$ of FRB. The deficiency of the faintest FRB (compared to the fitted Euclidean relation $N \propto S^{-3 / 2}$ ) may be attributable to inefficient detection of faint FRB or to cosmological effects. At somewhat higher fluences agreement is good, except for the anomalous Lorimer burst estimated (Lorimer, et al. 2007 ) to have $S=150 \mathrm{Jy}$ ms. The absence of FRB with 10 Jy ms $<S<150$ Jy ms casts doubt on the membership of the Lorimer burst in the FRB population.

$K(r) \rightarrow K$, a constant. More generally, $K(r)$ contains three powers of $1+z$ from the variation of the source density, minus one power from the redshift of the event rate and minus one power from the redshift of the photon energy (because fluence rather than flux is measured the rate of photon arrival does not contribute another factor). If the source spectrum is a power law $S \propto \nu^{-\gamma}$ then $K(r) \propto(1+z)^{1-\gamma}$. With $\gamma$ and the source evolution unknown, treating $K(r)$ as a constant may not be far wrong for $z \lesssim 1$. 
In the same spirit of approximation

$$
\begin{aligned}
d r & =\frac{c}{H_{0}} \frac{d z}{1+z} \\
r & =\frac{c}{H_{0}} \ln (1+z) \\
n_{e} & =n_{0}(1+z)^{3}=1.6 \times 10^{-7}(1+z)^{3} \mathrm{~cm}^{-3} \\
d \mathrm{DM}_{e f f} & =n_{e} d r=\frac{n_{0} c}{H_{0}}(1+z) d \ln (1+z) \\
\mathrm{DM}_{e f f} & =\frac{n_{0} c}{H_{0}}[(1+z)-1]
\end{aligned}
$$

where the baryons are assumed homogeneously distributed and ionized with the present-day density $n_{0}=1.6 \times 10^{-7} \mathrm{~cm}^{-3}$ and $\mathrm{DM}_{e f f}$ is the measured dispersion measure allowing for the higher frequency of the observed radiation in the distant universe. Then

$$
\frac{d N}{d \mathrm{DM}}=\frac{K c^{2}}{n_{0} H_{0}^{2}} \frac{[\ln (1+z)]^{2}}{1+z}
$$

Using Eq. 30 ,

$$
\frac{d N}{d \mathrm{DM}}=\frac{K c^{2}}{n_{0} H_{0}^{2}} \frac{\left[\ln \left(1+\frac{H_{0} \mathrm{DM}}{n_{0} c}\right)\right]^{2}}{1+\frac{H_{0} \mathrm{DM}}{n_{0} c}} .
$$

This is readily integrated

$$
N=\frac{K c^{3}}{3 H_{0}^{3}}\left[\ln \left(1+\frac{H_{0} \mathrm{DM}}{n_{0} c}\right)\right]^{3} \propto\left[\ln \left(1+\frac{\mathrm{DM}}{682 \mathrm{pc} \mathrm{cm}^{-3}}\right)\right]^{3} .
$$

Figure 4 shows the cumulative distribution of $N$ as a function of DM and a curve of the form of Eq. 33 with one fitted parameter $(K)$. The fit is good. The most striking feature is the absence, in both the data and the theoretical curve, of FRB with low dispersion measures. This is explained by the small volume $\propto z^{3}$ of space with low $z$; Euclidean geometry and the absence of cosmic evolution are good approximations at small redshifts. This supports the interpretation of the dispersion measures of FRB as resulting from propagation through intergalactic space, independent of any specific model of their origin. In contrast, models (Kulkarni, et al. 2014; Pen \& Connor 2015) that attribute FRB dispersion measures to quasi-static clouds (Section 5.2) in their vicinities make no specific predictions of the distribution of DM, but do not naturally explain the data shown in Fig. 4.

This result is robust against changes in the assumptions. For example, ignoring the $(1+z)^{-2}$ factor in $d \mathrm{DM}_{\text {eff }}$ that results from the from the $z$ dependence of the frequency of radiation along its path leads to a fit similar to that of Figure 4 (but with a different $K$ ). This robustness is not surprising, because the model is dominated by the $\propto z^{3}$ dependence of the volume and $N$ in the local (Euclidean) limit. 


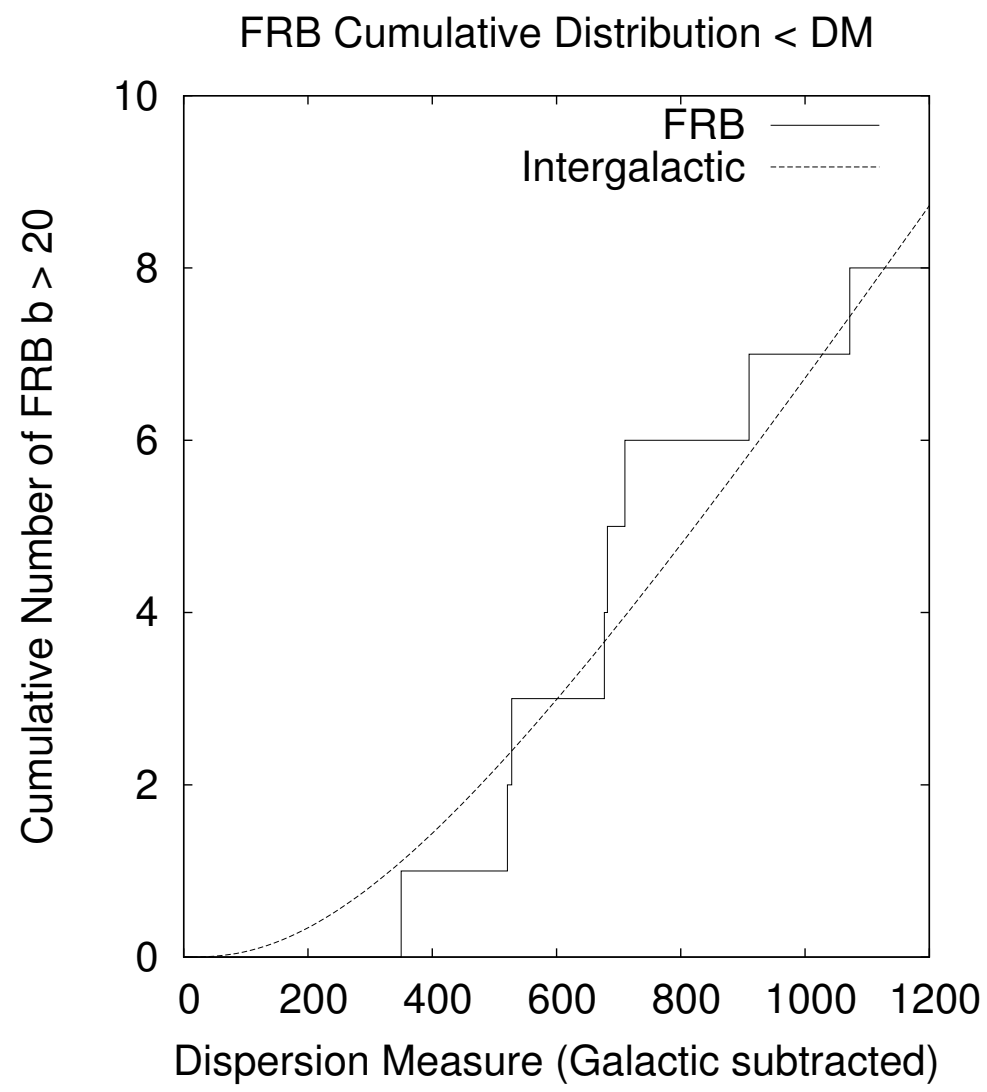

Fig. 4.- The cumulative distribution of FRB (the eight at high Galactic latitudes for which the Galactic contributions to their dispersion measures are small and can be reliably subtracted) vs. the extraGalactic part of their dispersion measures. The "Intergalactic" curve (Eq. 33) describes a simple model of dispersion attributable to the intergalactic medium. Agreement is good.

\section{Discussion}

Understanding of the high brightness emission of FRB may be as elusive as understanding of the emission of radio pulsars has been, nearly 50 years after their discovery. Radio pulsars were very quickly identified on the basis of their periods and slowing with rotating neutron stars. No such clues exist for FRB.

Even with only ten published FRB, and without a specific model of their sources, statistical arguments can constrain their origin, both physical and spatial. Their qualitative resemblance to radio pulsars, and in particular to the rare giant pulses of a few pulsars, suggests that they may be analogous events, writ large. Elementary energetic considerations show that this may be possible, but if so their sources must be very young neutron stars.

This leads to the hypothesis that, surrounded by young, dense, compact SNR, much of the 
dispersion measures of FRB may be attributed to those SNR Kulkarni, et al. 2014: Connor, Sievers \& Pen 2015). If so, the distances to FRB may be much closer than those indicated by attributing their dispersion measures to the intergalactic medium, relaxing the constraints on their energetics implied by the assumption of cosmological distances. This hypothesis predicts a specific form for the distribution of FRB dispersion measures. Unfortunately, comparison to the data (Fig. 2) shows a poor fit at both low and high dispersion measures. The former might be explained by termination of FRB activity as the neutron star spins down (or ages in some other manner), in analogy to the pulsar "death line", but the absence of FRB with very high dispersion measures is an argument against this origin of their dispersion.

The classic $\log N-\log S$ test, where $S$ is the FRB fluence rather than the (unmeasured) flux, may also be applied (Fig. 3). A fit to the relation $N \propto S^{-3 / 2}$ applicable to Euclidean geometry (cosmologically local sources) is adequate at intermediate values of $S$. A deficiency of low fluence FRB may be explained by sample incompleteness. If so, the fit at higher fluences indicates a source population with $z \lesssim 1$, but excludes a spatially bounded distribution such as the Galactic halo.

Similar conclusions may be drawn from the distribution of FRB dispersion measures less than a cutoff (Fig. 4). A simple model that includes both cosmologically local and distant sources (but that ignores the unknown evolution of the source population, and hence must be considered only qualitative for $z \gtrsim 1$ ) provides a good fit. In contrast to the similar inferences from the $\log N-\log S$ data, this conclusion does not depend on additional explanations of apparent deviations from the model. A robust conclusion, independent of any caveats applicable for $z \gtrsim 1$, is that the source population is homogeneously distributed in space at smaller $z$. This is an essentially Euclidean conclusion, but the satisfactory fit for $z \sim 1$ suggests that the distances are those inferred from the assumption that the dispersion measures are intergalactic. This is supported by the failure of the alternative expanding cloud (SNR) models of the dispersing matter (Fig. 2). Thee fact that this cosmological model of the distribution of dispersion measures is consistent with the data implies that nearly all the dispersion is intergalactic, in contrast with models (Kulkarni, et al. 2014 . Kulkarni, Ofek \& Neill 2015) in which a large portion of the dispersion measure is attributed to gas local to the FRB.

The extraordinarily intense Lorimer burst is not consistent with $N \propto S^{-3 / 2}$. The absence of weaker events was noted by Lorimer, et al. (2007), and indicates that it comes from a different population. With a fluence $\sim 100$ times the detection threshold, a homogeneous distribution in Euclidean space (its comparatively small dispersion measure indicates it must be cosmologically local) would suggest that $\sim 1000$ weaker events should have been detected, in contrast to the 10 actually detected, an anomaly that is significant at the $\approx 99 \%$ level (the detection of 10 weaker events implies a $1 \%$ probability of detecting one as strong as the Lorimer burst). If this was neither a statistical fluke (because of the need to reject anthropogenic transients, early searches may have had effective detection thresholds higher than their nominal thresholds) nor anthropogenic interference, it implies the Lorimer burst came from a distinct space-limited population whose local density far exceeds its mean cosmological density. Speculative possibilities include classes of intrinsically less 
luminous bursts in the Galactic halo or the Local Supercluster.

\section{A. Space Radar as a Source of Chirped Pulses?}

Since the discovery of FRB there has been concern that they might be anthropogenic, rather than astronomical, phenomena. This concern was exacerbated by the discovery by Burke-Spolaor, et al. (2011) of manifestly anthropogenic perytons, whose sources were recently identified by Petroff, et al. (2015b) as microwave ovens. Could FRB also be anthropogenic interference, perhaps chirped radar pulses? A ground-based radar might enter of sidelobes of a radio telescope, but would appear in all 13 Parkes beams, unlike the FRB. Hence we consider a radar on a satellite that might pass through a beam.

Radar systems may use chirped emission, compressed upon reception into narrow pulses, in order to obtain accurate range measurements without requiring excessive peak transmitted powers. The observation of FRB in a single beam at Parkes, in contrast to perytons (Burke-Spolaor, et al. 2011), indicates a distance $\gtrsim 20 \mathrm{~km}$, outside the first Fresnel zone, consistent with a radar satellite. There is no obvious reason for a radar to have a chirp $\omega \propto t^{-1 / 2}$ as observed, nor is there obvious reason not. However, the observed dispersed pulse durations of several tenths of a second would imply, for monostatic radar, target distances of at least half that light travel time to avoid interference of the transmission with the received scattered radiation. Such distances would be $\sim 10^{10} \mathrm{~cm}$, far beyond the range to plausible targets, and the return would be undetectably weak.

In contrast, bistatic radar can use arbitrarily long pulses. The pulse repetition intervals would have to have been longer than the lengths of time the radars were anywhere in the 13 beams of the Parkes Multibeam Pulsar Survey (about $0.3 \mathrm{~s}$ for a radar near the zenith in low Earth orbit), yet the pulse durations must have been shorter than the time required to cross a single beam. This explanation would also require at least as many radar satellites, each with a different chirp rate,

as FRB, because each FRB had a different dispersion measure, or satellites whose chirp rates were variable in some non-obvious manner. This combination of requirements makes the hypothesis of interference by an orbital chirped source implausible.

I thank J. Goodman and T. Piran for useful discussions, and an anonymous referee for pointing out the invalidity of an essential assumption of an earlier version of this paper.

\section{REFERENCES}

Burke-Spolaor, S., Bailes, M., Ekers, R., Marquart, J.-P. \& Crawford, F. III 2011 ApJ 727, 18.

Burke-Spolaor, S. \& Bannister, K. W. 2014 ApJ 792, 19.

Connor, L., Sievers, J. \& Pen, U.-L. 2015 arXiv:1505.05535. 
Dolag, K., Gaensler, B. M., Beck, A. M. \& Beck, M. C. 2015 MNRAS 451, 4277.

Eatough, R. P., Falcke, H., Karuppusamy, R. et al. 2013 Nature 501, 391.

Falcke, H. \& Rezzolla, L. 2014 A\&A 562, 137.

Hankins, T. H. \& Eilek, J. A. 2007 ApJ 670, 693.

Katz, J. I. 1982 ApJ 260, 371.

Katz, J. I. 1996 ApJ 463, 305.

Katz, J. I. 2014a Phys. Rev. D 89, 103009.

Katz, J. I. 2014b ApJ 788, 34.

Keane, E. F., Stappers, B. W., Kramer, M. \& Lyne, A. G. 2012 MNRAS 425, L71.

Keane, E. F. \& Petroff, E. 2015 MNRAS 447, 2852.

Krishnakumar, M. A., Mitra, D., Naidu, A., Joshi, B. C. \& Manoharan, P. K. 2015 arXiv:1501.05401.

Kulkarni, S. R., Ofek, E. O., Neill, J. D., Zhang, A. \& Juric, M. 2014 ApJ 797, 70.

Kulkarni, S. R., Ofek, E. O. \& Neill, J. D. 2015 arXiv:1511.09137.

Law, C. J., Bower, G. C., Burke-Spolaor, S. et al. 2014 arXiv:1412.7536.

Lee, L. C. \& Jokipii, J. R. 1975 ApJ 201, 532.

Lewandowski, W., Dembska, M., Kijak, J. \& Kowalińska, M. 2013 MNRAS 434, 69.

Lorimer, D. R., Bailes, M., McLaughlin, M. A., Narkevic, D. J. \& Crawford, F. 2007 Science 318, 777.

Luan, J. \& Goldreich, P. 2014 ApJ 785, L26.

Macquart, J.-P., \& Koay, J. Y. 2013 ApJ 776, 125.

Macquart, J.-P., Keane, E., Grainge, K. et al. 2015 arXiv:1501.07535.

Mereghetti, S. 2008 Astron. \& Ap. Rev. 15, 225.

Mori, K., Gotthalf, E. V., Zhang, S. et al. 2013 ApJ 770, L23.

Pen, U.-L. \& Connor, L. 2015 ApJ 807, 179.

Petroff, E., Bailes, M., Barr, E. D. et al. 2015a MNRAS 447, 246. 
Petroff, E., Johnston, S., Keane, E. F. et al. 2015b MNRAS 454, 457.

Ravi, V., Shannon, R. M. \& Jameson, A. 2015 ApJ 799, L5.

Rickett, B. J. 1977 ARA\&A 15, 479.

Sharon, K., Gal-Yam, A., Maoz, D., Filippenko, A. V. \& Guhathakurta, P., 2007 ApJ 660, 1165.

Soglasnov, V. A., Popov, M. V., Bartel, N., Cannon, W., Novikov, A. Y., Kondratiev, V. I. \& Altunin, V. I. 2004 ApJ 616, 439.

Spitler, L. G., Cordes, J. M., Hessels, J. W. T. et al. 2014b ApJ 790, 101.

Spitzer, L. Physics of Fully Ionized Gases 2nd ed. (Interscience, New York 1962).

Thompson, C. \& Duncan, R. C. 1992 ApJ 392, L9.

Thornton, D., Stappers, B., Bailes, M. et al. 2013 Science 341, 53.

Williamson, I. P. 1972 MNRAS 157, 55.

Zhou, B., Li, X., Wang, T., Fan, Y.-Z. \& Wei, D.-M. 2014 Phys. Rev. D 89, 107303. 